\title{
Improving the pollutant removal efficiency of packed-bed plasma reactors incorporating ferroelectric components
}

Ana Gómez-Ramírez ${ }^{1,2 *} \neq$, Antonio M. Montoro-Damas ${ }^{2} \neq$, Miguel A. Rodríguez ${ }^{3}$, Agustín R. González-Elipe ${ }^{2}$ and José Cotrino ${ }^{1,2^{*}}$

${ }^{1}$ Departamento de Física Atómica, Molecular y Nuclear, Universidad de Sevilla, Avda. Reina Mercedes, 41012 Sevilla, Spain.

${ }^{2}$ Laboratory of Nanotechnology on Surfaces. Instituto de Ciencia de los Materiales de Sevilla (CSIC-Universidad de Sevilla), Avda. Américo Vespucio 49, 41092 Sevilla, Spain.

${ }^{3}$ Instituto de Cerámica y Vidrio (CSIC), C/ Kelsen, 5, 28049 Madrid, Spain.

Corresponding Authors: Ana Gómez-Ramírez. E-mail: anamaria.gomez@icmse.csic.es, José Cotrino. E-mail: cotrino@us.es.

$\$$ These authors contributed equally.

\section{ABSTRACT}

In this work we have studied the plasma removal of air contaminants such as methane, chloroform, toluene and acetone in two parallel plate packed-bed dielectric barrier discharge 
(DBD) reactors of different sizes. Removal and energy efficiencies have been determined as a function of the residence time of the contaminated air within the reactor, the kind of packed-bed material (ferroelectrics or classical dielectric materials), the frequency and the incorporation of a ferroelectric plate onto the active electrode together with the inter-electrode ferroelectric pellets filling the gap. Results at low frequency with the small reactor and the ferroelectric plate showed an enhancement in energy efficiency (e.g., it was multiplied by a factor of six and three for toluene and chloroform, respectively) and in removal yield (e.g., it increased from $22 \%$ to $52 \%$ for chloroform and from $15 \%$ to $21 \%$ for methane). Such enhancements have been attributed to a higher energy of plasma electrons and a lower reactor capacitance found for this plate-modified configuration. A careful analysis of reaction efficiencies and electron energy distributions for the different investigated conditions and the simulation of the electric field at the necks between ferroelectric/dielectric pellets complete the present study. Overall, the obtained results prove the critical role of the barrier architecture and operating conditions for an enhanced performance of pollution removal processes using DBD systems.

KEYWORDS: Plasma decontamination, dielectric barrier discharge, ferroelectric materials, pollutant removal, VOC's removal, packed-bed plasma reactor.

\section{INTRODUCTION}

One of the most common applications of dielectric barrier discharge (DBD) plasmas is the decomposition removal of gaseous pollutants [1-4]. A great number of works have been published about the use of these plasma discharges for the removal of volatile organic compounds (VOCs) present in the air as a result of different industrial and in-door activities [5- 
18]. However, although a large variety of reactor set-ups and working conditions have been proposed to maximize the efficiency of these processes [19-23], most studies have addressed the kinetics of the removal process and paid little attention to the analysis of the plasma, the electrical operation parameters or the effect of the reactor configuration. Common working conditions in these works are a low concentration of pollutants in air, of the order of hundreds or, maximum, thousands ppms, and the pursuit of the full oxidation of VOCs to $\mathrm{CO}_{2}$ and $\mathrm{H}_{2} \mathrm{O}$ (other fully oxidized sub-products if the pollutant contains heteroatoms) with no formation of intermediate hazardous molecules.

Advanced arrangements of DBD reactors for gas reaction processes such as the reforming of hydrocarbons, $\mathrm{CO}_{2}$ conversion or the synthesis of ammonia incorporate a ferroelectric moderator between the metallic electrodes [24-28]. This design is advantageous with respect to classical configurations incorporating dielectric materials because the operating voltages can be smaller $[29,30]$ and high plasma currents can be maintained for relatively large separation gaps between electrodes [31, 32]. Among other factors, these improvements could be linked with the substantial amount of electrons that, emitted by polarized ferroelectric materials [33, 34], may contribute to increase the plasma current and to decrease the breakdown voltage. In this work we have used pellets of lead zirconate titanate (PZT), a rather seldom ferroelectric material when dealing with plasma DBD reactors. The study presents a fundamental study about the design of DBD reactors and the effect of ferroelectric materials on their performance for the abatement of VOCs and other hazardous gases like methane in air. For this purpose, a series of ferroelectric packed bed reactors with a parallel electrode configuration (most geometrical configurations in the literature use a cylindrical geometry and a two coaxial electrodes system $[12,15,16])$ have been used to test the influence of the electrode area (i.e., size of the reactor) and the effect of 
incorporating a ferroelectric plate together with the ferroelectric pellets filling the inter-electrode space. Although ferroelectric plates incorporated onto DBD electrodes have been demonstrated to be quite effective in reducing the ignition voltage in surface discharge plasmas [35], to our knowledge their incorporation together with pellets in a packed-bed reactor has not been intended up to now. This unprecedented trial has effectively demonstrated that these kind of mixed barriers render more efficient the packed-bed reactors. Besides analyzing the influence of the ferroelectric material distribution within the reactor, we have also studied the effect of reactant flow and other working parameters on the process efficiency. Finally, a comprehensive study of the electrical response of the reactor, both theoretically by means of the COMSOL software [36] and experimentally through the determination of the average electron energy and system capacitance has allowed us to correlate the efficiency of the decontamination processes with the architecture of the ferroelectric barrier and other operating parameters. Such an analysis has provided important clues to understand the system behavior and the possibilities for improvement and extrapolation to other reactor configurations. We think that the obtained results can be relevant to design more efficient packed-bed plasma reactors through the adjustment of the average energy of plasma electrons to values higher than the threshold required to effectively oxidize organic pollutant compounds in air.

\section{MATERIALS AND METHODS}

According to Figure 1a), the reactors utilized for the present investigation have a parallel plate configuration with a fixed gap space of $3 \mathrm{~mm}$. The inter-electrode space was filled with quasispherical pellets of PZT (lead zirconate titanate) with $1.25 \mathrm{~mm}$ of average diameter that were 
synthesized in our laboratory according to a complex process described in detail in [26] and that involves the sintering and sieving of pellets. The relative dielectric constant of PZT is approximately 1900 (APC International, LTD). The Curie point phase transformation of these ferroelectric pellets was $332{ }^{\circ} \mathrm{C}$ as determined by differential scanning calorimetry (DSC, TA Instruments Q600). The choice of this ferroelectric material instead of others like $\mathrm{BaTiO}_{3}$ more popular for DBD applications $[5,16]$ has been dictated by the prevalence of a high dielectric constant within a broader range of temperatures (although $\mathrm{BaTiO}_{3}$ dielectric constant can be as high as 10000 [37] it losses its ferroelectric behavior above $120^{\circ}$ ) and a higher reaction performance obtained for the ammonia synthesis with the same reactor filled with PZT [27]. To ascertain the convenience of using ferroelectrics as filling materials, some comparative abatement experiments were also carried out using similar size pellets of a dielectric material $\left(\mathrm{Al}_{2} \mathrm{O}_{3}\right.$, RGPBALLS S.r.1., Italy).

The active upper circle electrode was directly connected to the AC voltage supply, while the bottom electrode was grounded. In an alternative operating mode, a ferroelectric $\mathrm{LiNbO}_{3} 0.5 \mathrm{~mm}$ thick disk supplied by Roditi (Roditi International Corporation Ltd, England) was fixed onto this upper electrode (Figure 1b). These two-sides polished plates were cut at $128^{\circ}$ with respect to the $\mathrm{Y}$-axis and, as provided by the supplier, have the following main dielectric constants: $\mathrm{T}_{11}=85.2$, $\mathrm{T}_{22}=85.2$ and $\mathrm{T}_{33}=28.7$. The active metal and ferroelectric plate electrodes had diameters of 7.5 and $10 \mathrm{~cm}$, respectively. Further details about the geometrical characteristics of this reactor and working parameters and conditions can be found in previous publications reporting about the reforming of methane and the synthesis of ammonia [26, 27].

A bigger reactor with the same configuration and active electrode diameter of $30 \mathrm{~cm}$ was also tested. This scaled-up reactor, shown in Figure 1, was used to ascertain the influence of power 
density and other electrical parameters on the plasma removal efficiency. To properly compare the pollutant removal ratios of the two reactors, the gas residence time was set constant for the two systems by conveniently regulating the flow rates.

(a)

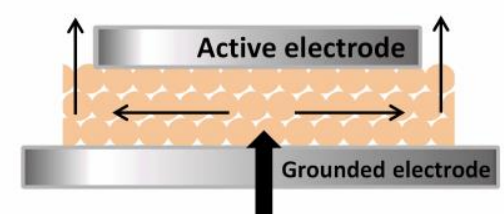

(b)

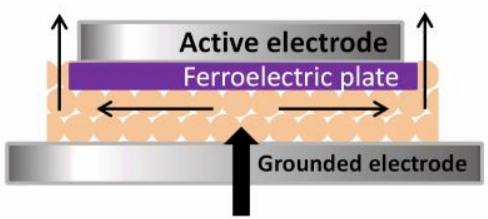

(c)

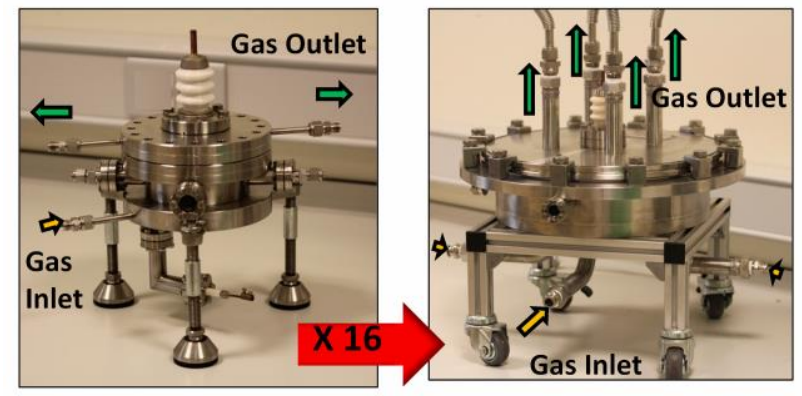

Figure 1. Schemes of the parallel plate reactors used in the present work: (a) Small reactor with ferroelectric pellets. (b) Small reactor with ferroelectric pellets and a $\mathrm{LiNbO}_{3}$ plate onto the active electrode. (c) Photographs of the small (left) and big size reactors (right), the latter had a 16 times bigger electrode area. Green and yellow arrows indicate the flow direction in each case. 
The three systems were fed with dry air (mixture of oxygen plus nitrogen with practically no residual $\mathrm{CO}_{2}$ ) as carrier gas and regulated concentrations of acetone, toluene and chloroform taken as examples of VOCs with, respectively, oxygen atoms, an aromatic structure and chlorine atoms. Methane has been also considered in this study because it is difficult to oxidize and can be taken as a reference for the removal of aliphatic hydrocarbons. The concentration of the VOCs in the air feeding the reactors was adjusted by mixing saturated air obtained by room temperature bubbling through these compounds in liquid form with different proportions of pure air. Methane gas was directly mixed with the carrier gas in the required proportion. Typical flow rates ranging from 100 to $1000 \mathrm{sccm}$ for the small reactor and $1600 \mathrm{sccm}$ for the bigger one were used for the experiments. The VOCs/methane concentrations, maintained constant for all experiments, were $2000 \mathrm{ppm}$ for both methane and acetone, and $3000 \mathrm{ppm}$ and $4000 \mathrm{ppm}$ for toluene and chloroform, respectively. These values are higher than typical concentrations of these pollutants in contaminated air or in other DBD experiments dealing with the abatement of pollutants $[5,19,23,25]$ because of the fundamental character of the present work aiming at improving design and operating conditions of reactors.

Removal efficiency was defined as the molar percentage of decomposed VOC/methane, while the energy efficiency was estimated as the amount of eliminated contaminant per consumed energy unit $(\eta, \mathrm{g} / \mathrm{kWh})$. We have preferred this definition of energy efficiency to the common "specific input energy" (J/L) parameter [3], because the latter relates the consumed energy to the total volume of gas passing through the reactor which, in the present experiments, refers to the air flow rather than to the amount of contaminants.

The removal efficiency was determined by mass spectrometry (Sensorlab, Prima Plus Pfeiffer Vacuum) following the decrease in pollutant peak intensities after igniting the plasma. 
Data were taken once the temperature (measured by using a thermocouple situated on the external wall of the reactors) of the small/big reactor walls stabilized after $30 \mathrm{~min}$ at a maximum value (up to $70^{\circ} \mathrm{C}$ when applying the highest powers utilized in each case). The error associated to the measurements was determined for four repeated experiments of the same decontamination process using the Student's $\mathrm{t}$ distribution. Within the limits of this technique, $\mathrm{CO}_{2}$ and $\mathrm{H}_{2} \mathrm{O}$ were the only products detected as a result of the removal process, indicating that this consisted in the total combustion of the pollutant molecules (neither $\mathrm{CO}$ nor other sub-products could be detected, see details about the evaluation of mass spectra in the supplementary material S1). A good correlation existed between the intensities of $\mathrm{CO}_{2}$ and $\mathrm{H}_{2} \mathrm{O}$ peaks and the decrease in the molecular fragments of the organic molecules after switching on the reactor. Minute amounts of $\mathrm{NO}$, attributed to the direct plasma reaction between oxygen and nitrogen, could be detected in some of the experiments. We attribute the absence of chlorine compounds in the experiments carried out with chloroform to their accumulation at the upmost surface layers of the ferroelectric pellets, as confirmed by surface analysis with XPS. The relatively high effective surface area of the ferroelectric pellets (BET specific surface of $0.74 \mathrm{~m}^{2} / \mathrm{g}$ ) assures very long operating times before saturation.

An amplifier (Trek, Model PD05034) connected to a function generator (Stanford Research System, Model DS345) was used to power the reactors. The applied signal waveform was always sinusoidal with frequencies set at $5 \mathrm{kHz}$ and $1 \mathrm{kHz}$. I(t) and $V(\mathrm{t})$ curves were monitored by means of an oscilloscope (Tektronix TDS2001C). Examples of these curves for an operating frequency of $5 \mathrm{kHz}$ are reported in Figure 2 for the small and scaled-up reactors at their stabilization temperatures. This figure also shows a simplified scheme of the electrical circuits utilized for the measurements. Experiments with the small reactor were carried out at a constant 
voltage of $3 \mathrm{kV}$. When it was modified with the ferroelectric plate, higher voltages were required to reach equivalent current values. The scaled-up reactor had to be operated at lower voltages to avoid short-circuits (see the result section). Therefore, to properly compare removal efficiencies in all reactors, all experiments were carried out utilizing similar current values. $V(t)$ and $I(t)$ were described by modified sinusoidal-like curves with a small phase shift (Figure 2 b) and c)) and no hints of microdischarges, as found in other DBD gas processes [3, 28, 38, 39, 40]. This difference must be attributed to the special design of our reactor set up (the short inter-electrode distance and the pellet size), the frequency used and the formation of homogenous plasma between the irregular ferroelectric pellets filling the inter-electrode space.

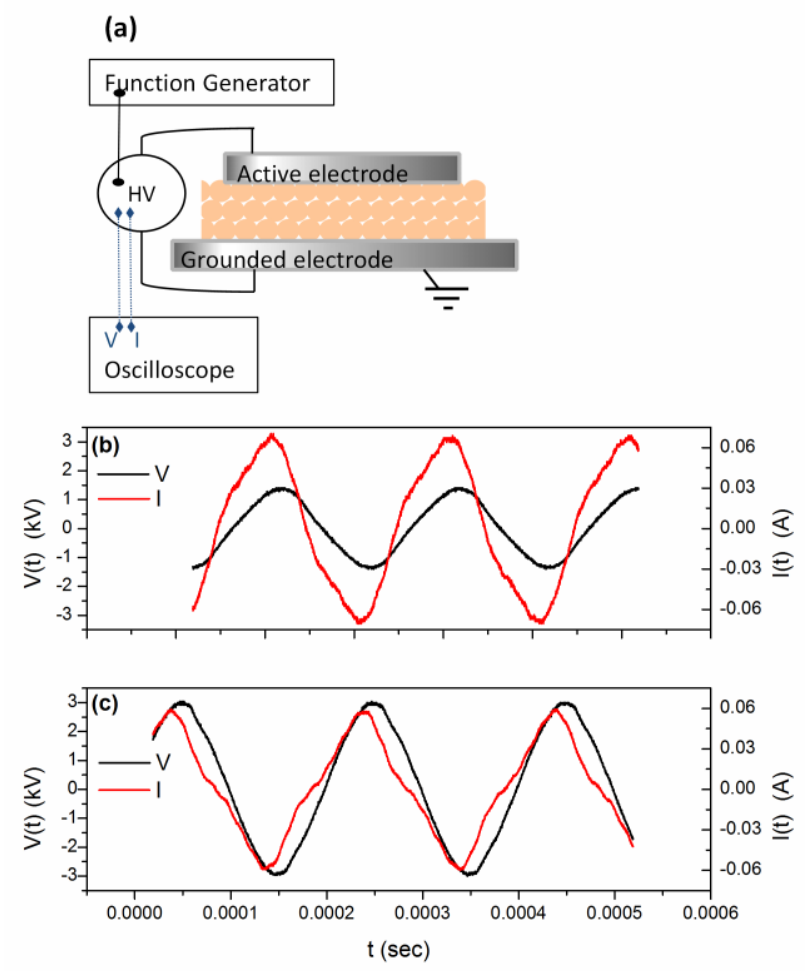


Figure 2. (a) Schematic of the electrical connections wiring used to activate and monitor the reactors operation. (b) $I(\mathrm{t})$ and $V(\mathrm{t})$ curves obtained at $5 \mathrm{kHz}$ for the scaled-up reactor, (c) idem for the small reactor. Gas mixture: dry air and methane.

The instantaneous accumulated charge on the electrode surface, $Q(\mathrm{t})$, was determined at any time by integration of $I(\mathrm{t})$. Lissajous plots calculated from $Q(\mathrm{t})$ and $V(\mathrm{t})$ curves were used to determine the consumed power, $P$, and the dielectric capacitance (i.e., capacitance of the dielectric material) $C_{d}(\mathrm{nF})$. The former is equivalent to the enclosed area within the plot and the latter can be calculated from its slope in the region corresponding to the active discharge phase, by assuming that the model proposed by Pipa et al. [41] is applicable to our system.

The current density, $J$, has been calculated as indicated in [42]:

$J=I /\left(\alpha^{*} A\right)$

where $A$ is the reactor electrode area, $\alpha$ the void fraction (by supposing a perfect packed-bed structure, $\alpha=0.09$ ) and $I$ the root mean squared value of the $I(\mathrm{t})$ curve. Equally, the power density, $P_{d}$, has been calculated taking into account the void fraction, $\alpha$ :

$P_{d}=\mathrm{P} /\left(\alpha^{*} A\right)$

where $P$ is the consumed power.

In high pressure discharges, plasma electron density can be approximated using the electron transport equation:

$n_{e}=J /\left(\mathrm{e}^{*} \mu_{e}^{*} E_{0}\right)$

where e is the electron charge, $E_{0}$ the electric field and $\mu_{e}$ the electron mobility approximated according to the Bolsig+ code solver [43]. Average electron energy, $\varepsilon_{0}$, was also estimated with the Bolsig+ code. $E_{0}$, the electric field strength in this equation, was calculated as $1 / 3\left(V_{\max } / d_{\max }\right)$, where $V_{\max }$ is the maximum value of the applied voltage, taken as the amplitude of $V(\mathrm{t})$, and $d_{\max }$ 
is the maximum inter-pellets distance taken as 0.7 times their average radius deduced by assuming their packing in the form of an ideal hexagonal compact structure. The fraction $1 / 3$ stems for the three plasma zone spaces defined by the two layers of ferroelectric pellets stacked in the inter-electrode space and through which the voltage drops (see supplementary material S2). This approximation clearly underestimates the actual value of electric field in necks and other smaller voids within the irregular packed bed of pellets, but provides reference values to follow their variation with the experimental conditions. To get a clearer qualitative insight of the electric field distribution between the ferroelectric pellets and between them and the electrodes, simulations were carried out using the COMSOL Multiphysics software [36]. That accounts for the electric field distribution through and between the pellets depending on their permittivity and relies on the resolution of the Poisson equation at the ferroelectric barriers present in the system in the absence of plasma. Dimensions and composition of ferroelectric materials used for these simulations are similar to the real ones in our reactor set-up.

\section{RESULTS AND DISCUSSION}

Safety of decomposition products, energy consumption and removal efficiency are three major concerns by the construction and operation of DBD reactors intended for decontamination purposes $[1-4,18,19,23]$. In this regard, incorporation of catalysts in the packed bed is a general strategy in order to favor the total oxidation of organic pollutants $[5,11,24]$. Since in the present study no significant traces of other byproducts apart from $\mathrm{CO}_{2}$ and $\mathrm{H}_{2} \mathrm{O}$ could be detected in the outlet gases, we assumed that the PZT pellets are acting as effective oxidation catalyst and that, therefore, the incorporation of an additional material is not necessary. In this regard, it is noteworthy the positive catalytic effect of PZT recently found by us for the DBD synthesis of 
ammonia [27]. Therefore, the present fundamental study focusses on the effect of reactor design and working conditions on the removal and energy efficiency of the decontamination processes.

\subsection{Removal efficiency}

In most works about the removal of air contaminants by means of packed-bed plasmas, the reactor configuration is cylindrical and no systematic studies are carried out varying geometry and/or reactor size $[6,9,12,15,24]$. In this section, we firstly report a comparison of process efficiency by varying the area of the electrodes for a parallel plate configuration. For the small reactor filled with PZT pellets, Figure 3 shows a compilation of $\mathrm{VOC} /$ methane removal efficiencies (in percentages) as a function of the residence time. A voltage of $3 \mathrm{kV}$ and a frequency of $5 \mathrm{kHz}$ were used for these experiments, conditions that rendered a current of $70 \mathrm{~mA}$ and a power density of $20.6 \mathrm{~W} / \mathrm{cm}^{2}$. For a flow rate of $100 \mathrm{sccm}$, practically $100 \%$ removal was obtained for acetone, chloroform and toluene while only 50\% was found for $\mathrm{CH}_{4}$. The decomposition efficiency always decreased for shorter residence times, although the evolution profile was dependent on the contaminant type: for toluene, acetone and chloroform it diminished rather slowly up to a residence time of $0.7 \mathrm{sec}$ and then sharply decreased for shorter times. Meanwhile, for methane it continuously decreased with this parameter. The two star points in the plot correspond to the scaled-up reactor working at $1.5 \mathrm{kV}, 5 \mathrm{kHz}$ and a similar operating current of $70 \mathrm{~mA}$, the maximum value achievable with this reactor before its electrical short-circuit. Since the big reactor could not be operated at higher voltages, this highest value of allowed current should entail the maximum achievable removal efficiency (to properly compare the results this also imposed to operate the small reactors at constant current). These conditions for the big reactor provided a power density of $0.6 \mathrm{~W} / \mathrm{cm}^{2}$ and a rather small removal percentage that clearly demonstrate its inefficiency. 


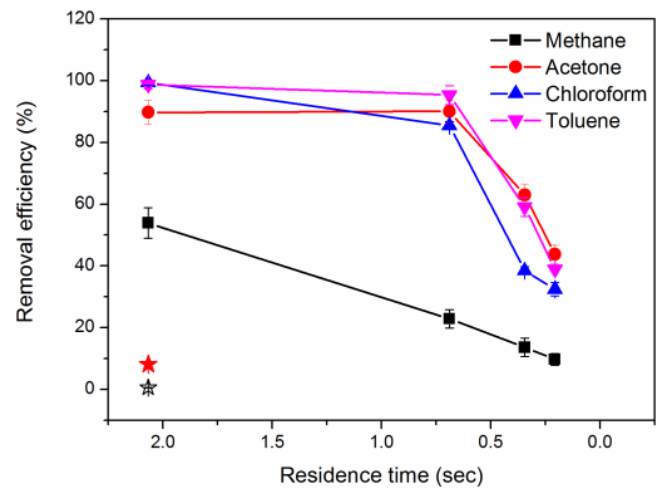

Figure 3. Representation of the percentages of $\mathrm{VOC} /$ methane removal as a function of the residence time for the four contaminants chosen for analysis. Data for the small (dots joined by a line) and scaled-up reactors (stars) are represented. Filled and unfilled-starts correspond to acetone and methane, respectively.

Most studies in the literature on packed-bed reactors for VOCs removal and other reactions use $\mathrm{BaTiO}_{3}$ or glass beads as moderator materials $[3,5,16,19,38,44]$, while works using less common ferroelectric materials are few [24, 27]. Choosing here PZT rather than $\mathrm{BaTiO}_{3}$ (with a permittivity as high as 1300 against 1900 for PZT) aimed at avoiding malfunctions in case the inner reactor temperature reaches the Curie phase transition temperature of $\mathrm{BaTiO}_{3}\left(120^{\circ} \mathrm{C}\right.$ as obtained by DSC), which is close to the $70^{\circ} \mathrm{C}$ experimentally determined at the reactor walls under steady state operating conditions. In our system a better performance of PZT pellets has been confirmed for other reactions [27]. To try determining the importance of using PZT as a ferroelectric material to enhance the process efficiency, we carried out a series of comparative experiments with the small reactor filled with $\mathrm{Al}_{2} \mathrm{O}_{3}$ pellets. Although the working parameters 
were the same than with the ferroelectric pellets, i.e., $5 \mathrm{kHz}$ and $3 \mathrm{kV}$, the measured current (i.e., $12.6 \mathrm{~mA}$ or $2.6 \mathrm{~W} / \mathrm{cm}^{2}$, the reactor underwent short-circuit for voltages higher than $3 \mathrm{kV}$ making impossible to make comparisons at constant power densities) was much lower in this case. The percentage of contaminant removal in these experiments was negligible: for example, no methane removal was observed for a residence time of 2 second. In agreement with other authors [42] we attribute this low efficiency to the low dielectric constant of $\mathrm{Al}_{2} \mathrm{O}_{3}$.

\subsection{Energy efficiency}

The operating frequency was of paramount importance in controlling the reactor performance. In general, decreasing the frequency at a fixed voltage rendered a smaller current density which, surprisingly, did not produce an equivalent decrease in either removal or energy efficiencies. This is clearly evidenced by the data in Table 1 comparing results for a residence time of 2 sec at two different frequencies, $5 \mathrm{kHz}$ (equivalent power density 20.6 of $\mathrm{W} / \mathrm{cm}^{2}$ ) and $1 \mathrm{kHz}$ (equivalent power density of $2.4 \mathrm{~W} / \mathrm{cm}^{2}$ ) and the same voltage of $3 \mathrm{kV}$. A first assessment from this comparison is that, although the percentage of conversion decreased at $1 \mathrm{kHz}(15 \pm 2 \%, 58 \pm$ $4 \%, 21.8 \pm 1.6 \%$ and $65 \pm 2 \%$ for $\mathrm{CH}_{4}, \mathrm{C}_{3} \mathrm{H}_{6} \mathrm{O}, \mathrm{CHCl}_{3}$ and $\mathrm{C}_{7} \mathrm{H}_{8}$, respectively) as compared with measurements at $5 \mathrm{kHz}$ (data in Figure 3), the energy efficiency $\eta$ was much higher when operating the small reactor at the lowest frequency.

Table 1. Energy efficiency data for different operating conditions and reactors

\begin{tabular}{|c|c|c|c|}
\hline \multirow{2}{*}{} & \multicolumn{3}{|c|}{ Energy efficiency, $\eta(\mathrm{g} / \mathrm{kWh})$} \\
\cline { 2 - 4 } & \multicolumn{2}{|c|}{ Small reactor } & Scaled-up reactor \\
\cline { 2 - 4 } & $5 \mathrm{kHz}$ & $1 \mathrm{kHz}$ & $5 \mathrm{kHz}$ \\
\hline $\mathrm{CH}_{4}$ & $0.070 \pm 0.006$ & $0.14 \pm 0.02$ & $0.021 \pm 0.003$ \\
\hline $\mathrm{C}_{3} \mathrm{H}_{6} \mathrm{O}$ & $0.38 \pm 0.02$ & $1.7 \pm 0.1$ & $1.0 \pm 0.1$ \\
\hline $\mathrm{CHCl}_{3}$ & $1.94 \pm 0.02$ & $3.0 \pm 0.2$ & - \\
\hline
\end{tabular}




\begin{tabular}{|c|c|c|c|}
\hline $\mathrm{C}_{7} \mathrm{H}_{8}$ & $0.87 \pm 0.01$ & $5.4 \pm 0.2$ & - \\
\hline
\end{tabular}

The data reported in this table confirmed the poor performance of the scaled-up reactor: only at 5 $\mathrm{kHz}$ and acetone, $\eta$ had a higher value, even though for a much lower removal efficiency in comparison with the small reactor (figure 3). We must stress that, since the residence time and contaminant concentration were maintained constant for the two reactors, the VOC/methane amount flowing per unit time through the scaled-up reactor was 16 times higher and similar efficiencies $\left(\eta\right.$ is defined as $\mathrm{g} \mathrm{kW}^{-1}$ ) would only be obtained for a 16 times higher removal efficiency in this case.

The bar diagram in Figure 4 reports the removal efficiencies obtained when the small reactor incorporated a ferroelectric plate at a fixed current of $13 \mathrm{~mA}$ and a power density of $2.8 \mathrm{~W} / \mathrm{cm}^{2}$ with the operating voltage fixed at $4 \mathrm{kV}$ and a frequency of $1 \mathrm{kHz}$. Clearly, the incorporation of a ferroelectric plate brought about a substantial increase of removal ratio, particularly for chloroform, acetone and toluene, and a net increase in energy efficiency that reached values of $0.16 \pm 0.01,2.05 \pm 0.08,5.7 \pm 0.3$ and $5.6 \pm 0.2 \mathrm{~g} / \mathrm{Kwh}$ for $\mathrm{CH}_{4}, \mathrm{C}_{3} \mathrm{H}_{6} \mathrm{O}, \mathrm{CHCl}_{3}$ and $\mathrm{C}_{7} \mathrm{H}_{8}$ respectively (i.e., much higher than those obtained without ferroelectric plate, see equivalent values in table 1). To our knowledge no similar approach combining ferroelectric plates and pellets has been reported in the literature. It is also noteworthy that the observed enhancements must be attributed to the ferroelectric nature of the plate: igniting the plasma required very high voltages in equivalent experiments performed with a dielectric plate (fused quartz), while the obtained low currents produced a negligible removal of pollutants. 


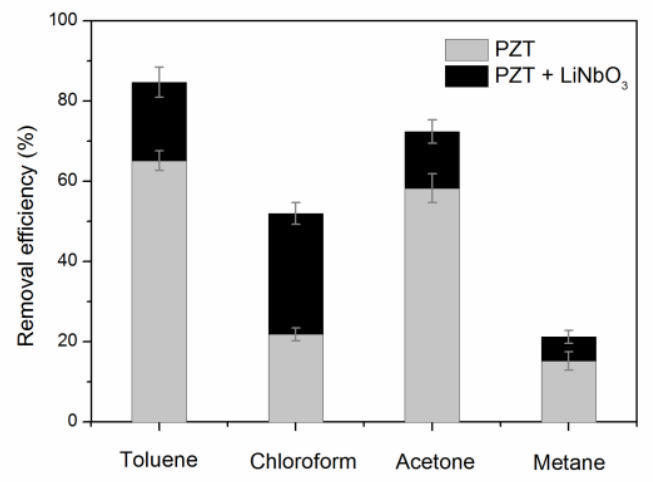

Figure 4. Comparison of the removal percentages of the studied contaminants decomposed in the small reactor with and without ferroelectric $\mathrm{LiNbO}_{3}$ plate (flow: $100 \mathrm{sccm}$ ).

In general, our results compare well with those of other recent works on packed-bed reactors. For example, similar removal efficiencies have been reported in the case of the acetone and toluene $[4,5,23,25]$, while for methane, our percentages of removal are higher than any other previously reported $[39,45]$. Recently, using a microhollow cathode discharge, Jiang et al. [46] have demonstrated a very high conversion rate for this contaminant (i.e., 80\%) when applying $0.5 \mathrm{kV}$ and a very low power. This result confirms a clear dependence between conversion and reactor size and suggests the possibility to increase the process efficiency by incorporating a ferroelectric layer in an array of small or microplasma DBD devices, as already intended with microhollow cathode devices working in parallel [47].

\subsection{Electrical characterization}

The reported removal efficiencies can be rationalized in terms of the electrical behavior of the reactor. Figure 5 shows a representation of modified Lissajous curves, expressed in the form of accumulated charge per unit area, for the scaled-up and small reactors operated according to the conditions indicated in Table 1. As explained in the experimental section, from these curves it is 
possible to estimate the system dielectric capacitance $C_{d}[41]$. The obtained values reported in Table 2 revealed the existence of an inverse correlation between energy efficiency (c.f. Table 1) and capacitance and that this latter magnitude decreased for the small reactor operated at lower frequencies and, even more, when inserting the ferroelectric plate. Since these measurements were carried out by applying similar currents, the lower capacitance found with plate suggests that the required higher voltage was contributing to increase both electron energy and removal efficiency. We will come back to this point in section 3.4.

Table 2. $C_{d}$ values for the selected operating conditions and reactors

\begin{tabular}{|l|l|}
\hline & $\begin{array}{l}C_{d} \\
(\mathrm{nF})\end{array}$ \\
\hline $5 \mathrm{kHz}$ & 1.6 \\
\hline $1 \mathrm{kHz}$ & 1.0 \\
\hline $1 \mathrm{kHz}+\mathrm{LiNbO}_{3}$ & 0.7 \\
\hline $\begin{array}{l}5 \mathrm{kHz} \\
\text { (Scaled-up reactor) }\end{array}$ & $13.5^{* *}$ \\
\hline
\end{tabular}

** A proper estimation of capacitance is not straightforward in this case due to the round shape of the Lissajous curve in Figure 5a, only a rough approximation can be used to render this value.

The Lissajous curves provided other interesting clues about the system performance [41]. For example, at a frequency of $5 \mathrm{kHz}$ the comparison of the scaled-up and small reactors in Figure 5(a) proves that in the latter case the maximum charge accumulated per surface area was significantly higher (i.e., 0.352 vs. $0.029 \mathrm{mC} / \mathrm{m}^{2}$ ), and a twice higher voltage was required to reach similar current values (i.e. $70 \mathrm{~mA}$ ). In agreement with other studies $[48,49]$, this difference indicates that the average electron energy, which must be proportional to both reduced electric field $E / n$ ( $E$ the electric field and $n$ the concentration of neutral particles) and applied voltage, was smaller in the scaled-up reactor. The Lissajous curves in Figure 5(b) corresponding to the 
small reactor with (at $1 \mathrm{kHz}$ ) and without (at 1 and $5 \mathrm{kHz}$ ) ferroelectric plate confirmed that the maximum applied voltage is higher with the plate, thus sustaining a higher average energy for the plasma electrons in this case. As previously suggested, we attribute this effect to a polarization enhancement of electric field in the vicinity of the plate [50]. In this regard, it is noteworthy that incorporating the ferroelectric plate did not modify the shape of the $I(\mathrm{t})$ and $V(\mathrm{t})$ curves, but imposed a higher voltage to keep constant the discharge current (see supplementary material S3).
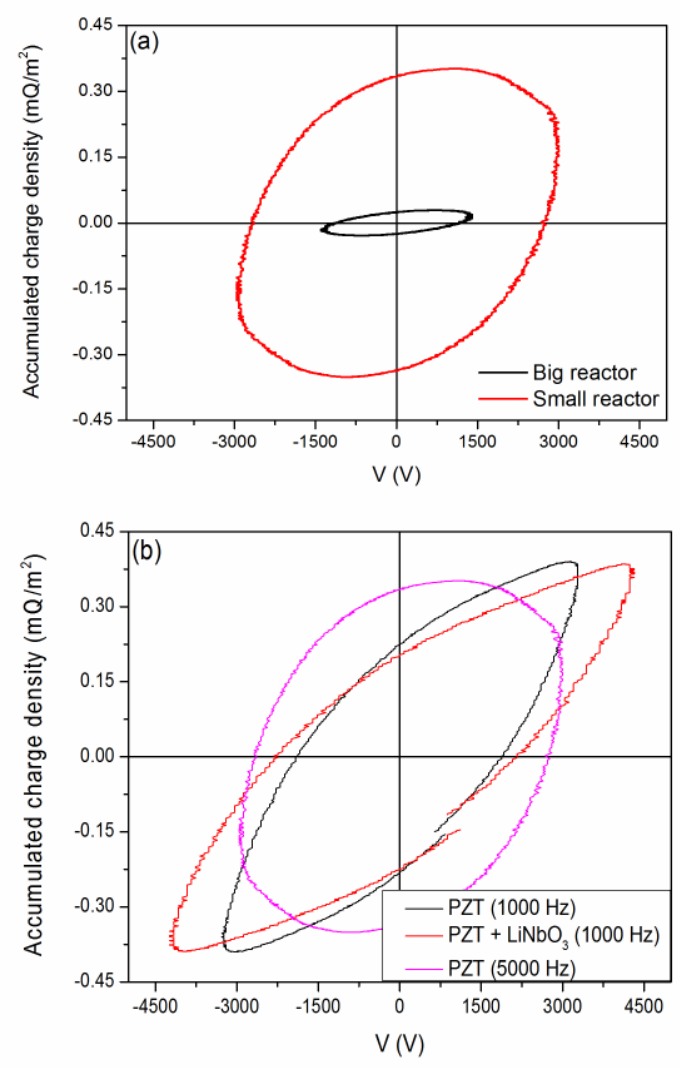

Figure 5. Lissajous curves expressed in terms of charge density (i.e. charge per electrode surface area) for the examined working conditions and reactors. (a) Scaled-up and small reactor working at $5 \mathrm{kHz}$. (b) Small reactor with and without ferroelectric plate working at the indicated frequencies. Gas mixture: dry air and methane. 
Figure $5 \mathrm{~b}$ shows a rounding of the Lissajous figure at higher frequency. We attribute this variation to changes in the dielectric constant of PZT with temperature [51-56] that would be particularly important at the highest frequency of $5 \mathrm{kHz}$, when the temperature of the reactor walls reached $70^{\circ} \mathrm{C}$ under steady state conditions (effects of the frequency itself on the dielectric constant are negligible $[52,57,58])$. Similar changes in the shape of the Lissajous curve have been previously reported by other authors and attributed to modifications in the resistive or capacitive behaviour of the reactor $[56,59]$.

\subsection{Electric field and electron density and energy}

Removal rates and energy efficiencies must depend on both plasma electron density and average electron energy. These parameters have been calculated as explained in the experimental section by using the Bolsig+ code for the different plasma reactor set-ups (i.e., small reactor with and without ferroelectric plate and scaled-up reactor) and pollutants. The values of the electric field $E_{0}$ used as an input parameter for the Bolsig+ code are reported in Table 3. Even if these values can only be considered for comparative purposes, they show that $E_{0}$ was higher for the small than for the scaled-up reactor and even higher when incorporating the ferroelectric plate in the small reactor.

Table 3. Calculated electric field, $E_{0}$, and electron energy determined by the Bolsig + code for the scaled-up and small reactor, in the latter case with and without ferroelectric plate.

\begin{tabular}{|c|c|c|}
\hline & $\begin{array}{c}E_{0}(\mathrm{~V} / \mathrm{m}) \\
\left(\cdot 10^{6}\right)\end{array}$ & $\varepsilon_{0}(\mathrm{eV})$ \\
\hline $5 \mathrm{kHz}$ & 2.3 & 3.1 \\
\hline $1 \mathrm{kHz}$ & 2.3 & 3.1 \\
\hline
\end{tabular}




\begin{tabular}{|c|c|c|}
\hline $1 \mathrm{kHz}+\mathrm{LiNbO}_{3}$ & 3.0 & 4.5 \\
\hline $\begin{array}{c}5 \mathrm{kHz} \\
\text { (Scaled-up reactor) }\end{array}$ & 1.1 & 1.3 \\
\hline
\end{tabular}

A more accurate estimation of the electric field distribution within the reactor has been carried out using the Comsol Multiphysics Software [36] by considering spherical beads. This software has been previously used by others to estimate the electrical field distribution in different systems [60-62], including packed-bed reactors [63]. In the present work, we have used this simulation procedure to figure out both the formation of hot spots between the ferroelectric pellets and the variations in the electric field when incorporating the $\mathrm{LiNbO}_{3}$ disc in the reactor (c.f., Figure 1 and 4). Figure 6 shows the results obtained for the small reactor with and without the ferroelectric plate. It is observed that the electric field reaches maximum values in the contact-points between pellets, further increasing by a $25 \%$ at these points when the ferroelectric plate is included in the calculations. A similar analysis for the electric displacement field, $D$, (see supplementary material S4) also reveals an increase in this magnitude at these "hot" contact points. Similar "hot spots" have also been identified for dielectric beads filling the inter-electrode space in packed-bed DBD discharges [63]. At this point it is important to mention that the $E_{0}$ values reported in Table 3, in the absence/presence of the ferroelectric plate, follow a similar trend than the distribution of electric field in Figure 6, although the actual values differ, e. g. at 3 $\mathrm{kV}$ without ferroelectric plate the calculated electric field in the hottest points is one order of magnitude higher than the reported $E_{0}$ value in Table 3 . Tentatively, to account for the data reported in Figure 4, we propose that the higher electric fields developed in the inter-pellet necks when incorporating the ferroelectric disc give rise to plasma electrons with a higher energy and therefore, more efficient for the plasma removal of VOCs. In this regard it is noteworthy that, as expected from its low dielectric constant, simulations carried out for an alumina packed-bed 
system revealed a very low electric field at the "hot points" (see supplementary material S5). This calculation agrees with the negligible removal efficiency observed with the reactor filled with pellets of this material.

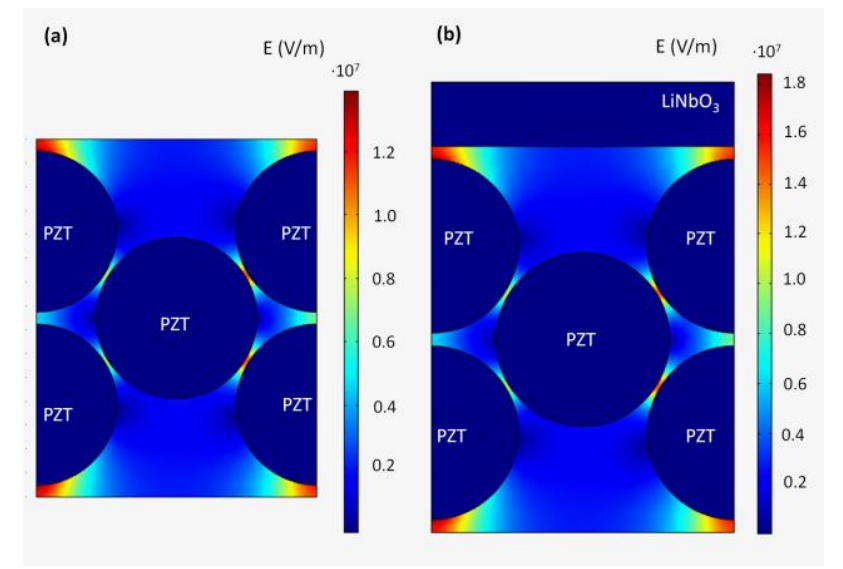

Figure 6. Electric field distribution for the un-modified (a) and modified small reactor (b). Input working parameters: (a) $\mathrm{V}=3 \mathrm{kV}$, (b) $\mathrm{V}=4 \mathrm{kV}$.

Table 3 summarizes the average electron energy values calculated with the Bolsig+ code. As suggested by the COMSOL calculations, the observed increase when incorporating the ferroelectric plate should be linked with local enhancements in the electric field at the hot spots formed between the ferroelectric pellets. Using similar simulation tools, the importance of these "hot-spots" has been demonstrated by Bogaerts et al. [50, 63, 64]. Similarly, by studying nitrogen plasmas, Takaki et al [42] showed that all the plasma parameters increase with the dielectric constant of the pellets and the applied voltage. Relying on these ideas, Kang et al. [32] claimed the importance of placing a catalyst in these inter-pellets regions.

Another important electrical magnitude for controlling plasma efficiencies is electron density that can also be estimated by using the Bolsig + code (see experimental section). The bar diagram presented in Figure 7 reveals that the smallest electron density was found at $5 \mathrm{kHz}$ for the scaledup reactor, followed by the small reactor modified with the ferroelectric plate and operated at 1 
$\mathrm{kHz}$ where, remarkably, the electron density was slightly smaller than in the unmodified small reactor. Roughly, these calculated electron densities follow the evolution of power densities utilized for the experiments, i.e., 20.6 and $2.4 \mathrm{~W} / \mathrm{cm}^{2}$ at 5 and $1 \mathrm{kHz}$ in the small reactor, and 0.6 $\mathrm{W} / \mathrm{cm}^{2}$ in the scaled-up one at $5 \mathrm{kHz}$. Taking together the electron density data and the energy efficiencies reported in Table 1, it appears that, despite the similar or even smaller electron density found in the small reactor with the ferroelectric plate, plasma electrons must be more efficient in activating the removal reactions. Tentatively, we propose that a higher electron energy promotes more effectively the activation of the pollutant decomposition processes. This hypothesis agrees with the qualitative assessments about electron energies deduced from the Lissajous curves and the average electron energies calculated using the Bolsig+ code and reported in Table 3 (we insist that these values should only be taken for comparison purposes, since they do not represent an exact evaluation of this magnitude, see experimental section). It is important to remark that the energies reported in table 3 are average values, meaning that in the tail of the electron energy distribution function electrons will have higher energies $(10 \mathrm{eV}$ or above). These electrons would be the responsible for the direct decomposition of VOCs through dissociation and/or electron excitation processes $[4,7]$. In addition, these high energy electrons would promote other processes like the direct dissociation of oxygen (energies in the order of 5.5 $\mathrm{eV}$ ) and the electron excitation of nitrogen molecule that would be preliminary steps for an effective oxidation of the pollutants (e.g., the former would intervene in a direct oxidative destruction of VOCs, while the latter could promote collision-induced dissociation of these molecules). Meanwhile, vibrational excitation processes, involving energies in the order of $1 \mathrm{eV}$ [65], would hardly promote the complete combustion removal of pollutants as observed in our case. 


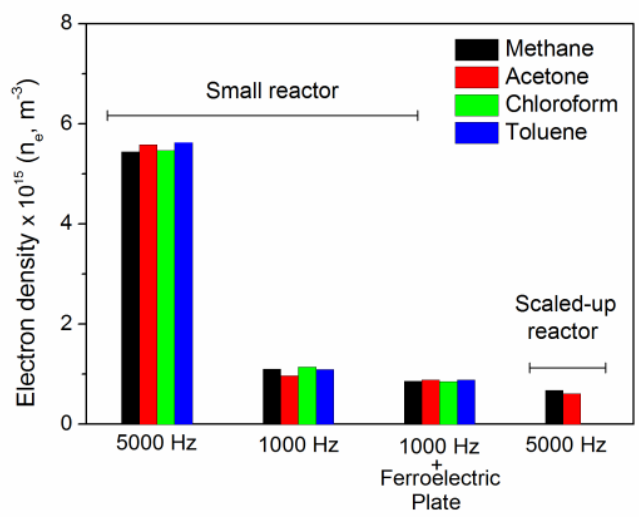

Figure 7. Calculated electron densities for different pollutants and reactor configurations as indicated in the plot (total flow: $100 \mathrm{sccm}$ ).

\section{CONCLUSIONS}

The previous results and discussion have shown that packed bed parallel plate DBD reactors are quite efficient for the removal of VOCs and other contaminants (results are shown for acetone, toluene, chloroform or methane) in air. They have also proved that a parallel plate design permits to assess the effect of increasing the active discharge area, and therefore the reactor size, without affecting other working parameters such as distance between electrodes or gas flow distribution. In this regard, an important conclusion of the work has been that efficiency comparatively decreases for larger electrodes mainly because the dielectric capacitance is relatively higher for big reactors. Other important conclusions refer to characteristics of the packed bed that contribute to increase the energy and removal efficiencies of the system. Firstly, substituting the pellets of a dielectric by a ferroelectric material has proved to be essential to improve the system performance. Secondly, we have found that fixing a ferroelectric plate onto the active metallic electrode substantially contributes to increase the average energy of the plasma electrons, resulting in a smaller system capacitance and an enhancement of reactor 
performance. The appearance of plasma "hot" spots between the ferroelectric pellets with a high electron density and energy has revealed crucial to enhance the overall performance of the discharges in ferroelectric packed bed reactors.

\section{ACKNOWLEDGMENT}

This work has been carried out thank to the financial support of the Junta de Andalucía (project P12-FQM-2265) and the MINECO-CSIC (project RECUPERA 2020). One of the authors, A. Gómez-Ramírez acknowledges financial support from MINECO (Spain) through the "Formación Postdoctoral 2013”.

\section{REFERENCES}

[1] U. Kogelschatz, Dielectric-Barrier Discharges: Their History, Discharge Physics, and Industrial Applications, Plasma Chem. Plasma Process. 23 (2003) 1-46.

[2] H. Kim, Nonthermal Plasma Processing for Air-Pollution Control: A Historical Review, Current Issues, and Future Prospects, Plasma Process. Polym. 1 (2004) 91-110.

[3] T. Zhu, VOCs Removal Using the Synergy Technology Basing on Nonthermal Plasma Technology, in: N. Mazzeo (ed.), Chemistry, Emission Control, Radioactive Pollution and Indoor Air Quality, InTech, 2011, pp. 81-132.

[4] M. Bahri, F. Haghighat, Plasma-Based Indoor Air Cleaning Technologies: The State of the Art-Review, Clean - Soil, Air, Water 42 (2014) 1-14.

[5] A. M. Vandenbroucke, R. Morent, N. De Geyter, C. Leys, Non-thermal plasmas for noncatalytic and catalytic VOC abatement, J. Hazard. Mater. 195 (2011) 30-54. 
[6] T. Oda, Non-Thermal Plasma Processing for Environmental Protection: Decomposition of Dilute VOCs in Air, J. Electrostat. 57 (2003) 293-311.

[7] M. P. Cal, M. Schluep, Destruction of benzene with non-thermal plasma in dielectric barrier discharge reactors, Environ. Prog. Sustain. Energy 20 (2001) 151-156.

[8] T. Oda, A. Kumada, K. Tanaka, T. Takahashi, S. Masuda, Low-temperature atmosphericpressure discharge plasma processing for volatile organic compounds, J. Electrostat. 35 (1995) 93-101.

[9] A. Ogata, K. Mizuno, S. Kushiyama, T. Yamamoto, Methane decomposition in a barium titanate packed-bed nonthermal plasma reactor, Plasma Chem. Plasma Process. 18 (1998) 363373.

[10] C. Fitzsimons, F. Ismail, J. C. Whitehead, J. J. Wilman, The chemistry of dichloromethane destruction in atmospheric-pressure gas streams by a dielectric packed-bed plasma reactor, J. Phys. Chem. A 104 (2000) 6032-6038.

[11] Y. Li, Z. Fan, J. Shi, Z. Liu, J. Zhou, W. Shangguan, Removal of Volatile Organic Compounds (VOCs) at Room Temperature Using Dielectric Barrier Discharge and PlasmaCatalysis, Plasma Chem. Plasma Process. 34 (2014) 801-810.

[12] L. Jiang, R. Zhu, Y. Mao, J. Chen, L. Zhang, Conversion Characteristics and Production Evaluation of Styrene/o-Xylene Mixtures Removed by DBD Pretreatment, J. Environ. Res. Public Health 12 (2015) 1334-1350.

[13] B. Jiang, J. Zheng, S. Qiu, M. Wu, Q. Zhang, Z. Yan, Q. Xue, Review on electrical discharge plasma technology for wastewater remediation, Chem. Eng. J. 236 (2014) 348-368. 
[14] C. W. Parka, J. Hwanga, Susceptibility constants of airborne bacteria to dielectric barrier discharge for antibacterial performance evaluation, J. Hazard. Mater. 244-245 (2013) 421-428.

[15] M. Takayama, K. Ebihara, H. Stryczewska, T. Ikegami, Y. Gyoutoku, K. Kubo, M. Tachibana, Ozone generation by dielectric barrier discharge for soil sterilization, Thin Solid Films 506-507 (2006) 396-399.

[16] T. Kuwahara, M. Okubo, T. Kuroki, H. Kametaka, T. Yamamot, Odor Removal Characteristics of a Laminated Film-Electrode Packed-Bed Nonthermal Plasma Reactor, Sensors $11(2011)$ 5529-5542.

[17] I. Stasiulaitiene, D. Martuzevicius, W. Abromaitis, M. Tichonovas, J. Baltrusaitis, R. Brandenburg, A. Pawelec, A. Schwock, Comparative life cycle assessment of plasma-based and traditional exhaust gas treatment technologies, J. Clean. Prod. 112 (2016) 1804-1812.

[18] T. Matsumoto, D. Wang, T. Namihira, H. Akiyama, Non-Thermal Plasma Technic for Air Pollution Control, in: B. Haryanto (ed.), Air Pollution - A Comprehensive Perspective, InTech, 2012, pp. 2015-234.

[19] A. M. Harling, D. J. Glover, J. C. Whitehead, K. Zhang, Industrial scale destruction of environmental pollutants using a novel plasma reactor, Ind. Eng. Chem. Res. 47 (2008) 58565860.

[20] A. A. Assadi, A. Bouzazaand, D. Wolbert, Treatment of Gaseous Effluents by Using Surface Discharge Plasma in Continuous Reactors: Process Modelling and Simulation, Can. J. Chem. Eng. 93 (2015) 206-112. 
[21] H. Zhang, K. Li, C. Shu, Z. Lou, T. Sun, J. Jia, Enhancement of styrene removal using a novel double-tube dielectric barrier discharge (DDBD) reactor, Chem. Eng. J. 256 (2014) 107118.

[22] K. Takaki, M. Shimizu, T. Sasaki, S. Kato, S. Mukaigawa, T. Fujiwara, Effect of Electrode Shape in Dielectric Barrier Discharge Plasma Reactor for NOx Removals, IEEE International Conference on Plasma Science 2003, 317.

[23] G. Xiao, W. Xu, R. Wu, M. Ni, Ch. Du, X. Gao, Z. Luo, K. Cen, Non-Thermal Plasmas for VOCs Abatement, Plasma Chem. Plasma Process. 34 (2014) 1033-1065.

[24] F. Holzer, F. D. Kopinke, U. Roland, Influence of Ferroelectric Materials and Catalysts on the Performance of Non-Thermal Plasma (NTP) for the Removal of Air Pollutants, Plasma Chem. Plasma Process. 25 (2005) 595-611.

[25] Ch. Zheng, X. Zhu, X. Gao, L. Liu, Q. Chang, Z. Luo, K. Cen, Experimental study of acetone removal by packed-bed dielectric barrier discharge reactor, J. Ind. Eng. Chem. 20 (2014) 2761-2768.

[26] A. M. Montoro de Damas, J. J. Brey, M. A. Rodríguez, A. R. González-Elipe, J. Cotrino, Plasma reforming of methane in a tunable ferroelectric packed-bed dielectric barrier discharge reactor, J. Power Sources 296 (2015) 268-275.

[27] A. Gómez-Ramírez, J. Cotrino, R. M. Lambert, A. R. González-Elipe, Efficient synthesis of ammonia from $\mathrm{N}_{2}$ and $\mathrm{H}_{2}$ alone in a ferroelectric packed-bed DBD reactor, Plasma Sources Sci. Technol. 24 (2015) 065011-65017. 
[28] D. Mei, X. Zhu, Y.-L. He, J. D. Yan, X. Tu, Plasma-assisted conversion of $\mathrm{CO}_{2}$ in a dielectric barrier discharge reactor: understanding the effect of packing materials, Plasma Sources Sci. Technol. 24 (2015) 015011-015021.

[29] A. Gómez-Ramírez, V. J. Rico, J. Cotrino, A. R. González-Elipe, R. M. Lambert, Low Temperature Production of Formaldehyde from Carbon Dioxide and Ethane by Plasma-Assisted Catalysis in a Ferroelectrically Moderated Dielectric Barrier Discharge Reactor, ACS Catal. 4 (2014) 402-408.

[30] V. J. Rico, J. L. Hueso, J. Cotrino, A. R. González-Elipe, Evaluation of different dielectric barrier discharge plasma configurations as an alternative technology for green $\mathrm{C} 1$ chemistry in the carbon dioxide reforming of methane and the direct decomposition of methanol, J. Phys. Chem. A 114 (2010) 4009-4016.

[31] V. J. Rico, J. L. Hueso, J. Cotrino, V. Gallardo, B. Sarmiento, J. J. Brey, A. R. GonzálezElipe, Hybrid catalytic-DBD plasma reactor for the production of hydrogen and preferential CO oxidation (CO-PROX) at reduced temperatures, Chem. Commun. (2009) 6192-6194.

[32] W. S. Kang, J. M. Park, Y. Kim, S. H. Hong, Numerical study on influences of barrier arrangements on dielectric barrier discharge characteristics, IEEE Trans. Plasma Sci. 31 (2003) $504-510$.

[33] H. W. Gundel, High-intense pulsed electron emission by fast polarization changes in ferroelectrics, Ferroelectrics 184 (1996) 89-98.

[34] J. F. Scott, Application of Modern Ferroelectrics, Science 315 (2007) 954-959 
[35] M. J. Johnson, D. B. Go, Ferroelectric crystals for the low-voltage operation of surface dielectric barrier discharges, Appl. Phys. Lett. 105 (2014) 264102.

[36] Comsol Multiphysics Modelling Software, www.comsol.com.

[37] K. Jogan, A. Mizuno, T. Yamamoto, J-Sh. Chang, The Effect of Residence Time on the $\mathrm{CO}_{2}$ Reduction from Combustion Flue Gasses by an AC Ferroelectric Packed Bed Reactor, IEEE Trans. Ind. Appl. 29 (1993) 876-881.

[38] W. Liang, J. Li, J. Li, Y. Jin, Abatement of toluene from gas streams via ferro-electric packed bed dielectric barrier discharge plasma, J. Hazard. Mater. 170 (2009) 633-638.

[39] H. J. Gallon, X. Tu, J. Ch. Whitehead, Effects of Reactor Packing Materials on $\mathrm{H}_{2}$ Production by $\mathrm{CO}_{2}$ Reforming of $\mathrm{CH}_{4}$ in a Dielectric Barrier Discharge, Plasma Process. Polym. 9 (2012) 90-97

[40] Akira Mizuno and Hiroshi Ito, Basic performance of an electrostatically augmented filter consisting of a packed ferroelectric pellet layer, J. Electrostat. 25 (1990) 97-107.

[41] A. V. Pipa, T. Hoder, J. Koskulics, M. Schmidt, R. Brandenburg, Experimental determination of dielectric barrier discharge capacitance, Rev. Sci. Instrum. 83 (2012) $075111-$ 075116.

[42] K. Takaki, J. S. Chang, K. G. Kostov, Atmospheric Pressure of Nitrogen Plasmas in a Ferro-electric Packed Bed Barrier Discharge Reactor Part I: Modeling, IEEE Trans. Dielectr. Electr. Insul. 11 (2004) 481-490. 
[43] G. J. M. Hagelaar, L .C. Pitchford, Solving the Boltzmann equation to obtain electron transport coefficients and rate coefficients for fluid models, Plasma Sources Sci. Technol. 14 (2005) 722, http://nl.lxcat.net/solvers/BOLSIG+/.

[44] A. Ogata, D. Ito, K. Mizuno, S. Kushiyama, T. Yamamoto, Removal of Dilute Benzene Using a Zeolite-Hybrid Plasma Reactor, IEEE Trans. Ind. Appl. 37 (2001) 959-964.

[45] K. J. Pringle, J. C. Whitehead, J.J. Wilman, J. H. Wu, The chemistry of methane remediation by a non-thermal atmospheric pressure plasma, Plasma Chem. Plasma Process. 24 (2004) 421-434.

[46] Ch. Jiang, A.-A. H. Mohamed, R. H. Stark, J. H. Yuan, K. H. Schoenbach, Removal of Volatile Organic Compounds in Atmospheric Pressure Air by Means of Direct Current Glow Discharges, IEEE Trans. Plasma Sci. 33 (2005) 1416-1425.

[47] J. G. Eden, S. J. Park, N. P. Ostrom, S. T. McCain, C. J. Wagner, B. A. Vojak, J. Chen, C. Liu, P. von Allmen, F. Zenhausern, Microplasma devices fabricated in silicon, ceramic, and metal/polymer structures: arrays, emitters and photodetectors, J. Phys. D: Appl. Phys. 36 (2003) 2869-2877.

[48] S. Mohanty, S. P. Das, G. Sahoo, R. Paikaray, P. S. Das, S. Samantaray, D. S. Patil, Effect on Plasma Parameters in a Dielectric Barrier Discharge Reactor with Volatile Organic Compounds, Proceedings of the International Conference on Plasma Science and Applications (ICPSA-2014), Vol. 10, No. II, December, 2014, pp 24-33.

[49] U. Kogelschatz, B. Eliasson, W. Egli, Dielectric-Barrier Discharges. Principle and Applications. Journal de Physique IV Colloque, 1997, 07 (C4), pp.C4-47-C4-66. 
[50] K. V. Laer, A. Bogaerts, Improving the conversion and energy efficiency of carbon dioxide splitting in a zirconia-packed dielectric barrier discharge reactor, Energy Technol., 3, (2015) 1038-1044.

[51] Q. M. Zhang, H. Wang, N. Kim, L. E. Cross, Direct evaluation of domain-wall and intrinsic contributions to the dielectric and piezoelectric response and their temperature dependence on lead zirconate-titanate ceramics, J. Appl. Phys. 75 (1994) 454-459.

[52] Properties of PZT-Based Piezoelectric Ceramics Between- 150 and $250^{\circ} \mathrm{C}, \mathrm{M}$. W. Hooker, Nasa Report - NASA / CR- 1998-208708, 1998.

[53] C. Miclea, C. Tanasoiu, L. Amarande, C. F. Miclea, C. Plavitu, M. Cioangher, L. Trupina, C. T. Miclea, C. David, Effect of Temperature on The Main Piezoelectric Parameters of A Soft PZT Ceramic, Rom. J. Inf. Sci. Tech. 10 (2007) 243-250.

[54] T. Singh, A. Kumar, U. C. Naithani, Field dependent dielectric study of $\mathrm{PbTiO}_{3}$ and $\mathrm{KNbO}_{3}$ ferroelectric perovskites, Indian J. Pure \& Appl. Phys. 48 (2012) 47-51.

[55] L. Jin, PhD thesis, Broadband Dielectric Response in Hard and Soft PZT: Understanding Softening and Hardening Mechanisms, THĖSE NO 4988, École Polytechnique Fédérale De Lausanne, Lausanne 2011.

[56] A. M. Harling, H-H. Kim, S. Futamura, J. Ch. Whitehead, Temperature Dependence of Plasma-Catalysis Using a Nonthermal, Atmospheric Pressure Packed Bed; the Destruction of Benzene and Toluene, J Phys. Chem C, 111 (2007) 5090-5095.

[57] S. K. Han, S. J. Lee, J. Kim, K.-Y. Kang, Measurement of Microwave Dielectric Constant for Ferroelectric $\mathrm{Pb}(\mathrm{Zr}, \mathrm{Ti}) \mathrm{O}_{3}$ Thin Films, J. Korean Phys. Soc. 32 (1998) S364-S366. 
[58] E. M. Bourim, H.-Y. Kim, J.-S. Yang, J.-W. Yang, K.-S. Woo, J.-H. Song, S.-K. Yun, Creep behavior of undoped and $\mathrm{La}-\mathrm{Nb}$ codoped PZT based micro-piezoactuators for microoptical modulator applications, Sensor. Actuat. A-Phys. 155 (2009) 290-298.

[59] H-H. Kim, Y. Teramoto, A. Ogata, H. Takagi, T. Nanba, Plasma Catalysis for Environmental Treatment and Energy Applications, Plasma Chem. Plasma Process. 36 (2016) 45-72.

[60] K. -S. Shin, J. H. Ji, K. S. Hwang, S. Ch. Jun, J. Y. Kang, Sensitivity Enhancement of Bead-based Electrochemical Impedance Spectroscopy (BEIS) biosensor by electric fieldfocusing in microwells, Biosens. Bioelectron. 85 (2016) 16-24.

[61] M. Li, H. Liu, Z. Ning, J. Ren, H. Tang, D. Yu, E.V. Demidov, S. I. Eliseev, A. A. Kudryavtsev, 2-D Extended Fluid Model of Applied-Field Magnetoplasmadynamic Thruster with Solid and Hollow Cathodes, IEEE Trans. Plasma Sci.43 (2015) 4034-4042.

[62] X. Liu, C. Liu, J. Zou, Similarities and differences of AC/DC arc plasma characteristics in vacuum interrupter, Chinese J. Vac. Sci. Technol., 35 (2015) 1203-1208.

[63] K. V. Laer, A. Bogaerts, Fluid modelling of a packed bed dielectric barrier discharge plasma reactor, Plasma Sources Sci. Technol. 25 (2016) 015002.

[64] K. V. Laer, St. Kolev, A. Bogaerts, Modelling of a packed bed dielectric barrier discharge plasma reactor, 22nd International Symposium on Plasma Chemistry, Book of abstracts 2015, Antwerp, Belgium.

[65] A. Fridman, Plasma Chemisty, first ed., Cambridge University Press, New York, 2008. 